INTERVENTIONAL CARDIOLOGY AND SURGERY

\title{
Detection of coronary microembolisation by Doppler ultrasound during percutaneous coronary interventions
}

\author{
P Bahrmann, H R Figulla, M Wagner, M Ferrari, A Voss, G S Werner
}

Heart 2005:91:1186-1192. doi: 10.1136/hrt.2004.048629

See end of article for authors' affiliations

Correspondence to: Dr Philipp Bahrmann, Clinic of Internal Medicine I, Friedrich-Schiller Úniversity, Erlanger Allee 101, 07740 Jena, Germany; philipp. bahrmann@med.uni-jena. de

Accepted

26 November 2004
Objective: To validate an intracoronary Doppler ultrasound device for high intensity transient signals (HITS) detection and to assess the incidence of HITS during percutaneous coronary intervention (PCI). Methods and Results: In an in vitro model, particle count and number of HITS detected by an intracoronary 0.014 inch Doppler wire were closely correlated $(r=0.97, p<0.001)$. In the clinical study, 32 patients (mean (SD) age 61 (11) years; 23 men, nine women) with coronary artery disease were treated with balloon dilatation and stent implantation for a single vessel stenosis. In these patients HITS were detected during $\mathrm{PCl}$ in $84 \%$ (27 of 32). Reproducibility $(r=0.99, p<0.001)$ and interobserver agreement $(r=0.84, p<0.001)$ of HITS counts were significant. The number of HITS after stent implantation was significantly higher than after balloon dilatation (1 1 (7) $v 2$ (4), $\mathrm{p}<0.001$ ). Postprocedural coronary flow velocity reserve (CFVR) was $<2.0$ in $55 \%$ (16 of 29) of all patients after balloon dilatation and $<2.0$ in $23 \%$ (six of 26 ) after stent implantation. The number of HITS after stent implantation did not differ significantly between patients with CFVR $<2.0$ and patients with CFVR $\geqslant 2.0$ (12 (8) v 10 (7), not significant).

Conclusions: Embolic particles can be detected as HITS by an intracoronary Doppler ultrasound device. Coronary microembolism is often observed during PCl, especially after stent implantation. However, the incidence of HITS alone does not explain a reduced CFVR after PCI.
A fter percutaneous coronary intervention (PCI), a reduced coronary flow velocity reserve (CFVR) is often observed, often associated with an increase of cardiac marker enzymes. Creatine kinase (CK) or CK-MB isoenzyme after PCI was reported to be increased in $3-30 \%$ of patients after $\mathrm{PCI}^{1-4}$ and troponin I or T was increased even more often in $30-40 \%{ }^{5-7}$ The rate of periprocedural increase of CK and troponin induced by stenting appeared to be greater than that after balloon angioplasty. ${ }^{8}$ The clinical implications of small troponin rises after PCI are not yet determined. but periprocedural CK-MB increases have been associated with a high risk of adverse cardiac events. ${ }^{3} 9$

The pathogenesis of the enzyme increase has been the subject of recent controversy. Microvascular obstruction by inflammation, oedema, reperfusion injury, and coronary microembolisation are likely explanations..$^{10}$ New devices, which provide distal protection during transcatheter treatment of aortocoronary saphenous vein graft stenosis, have supported the concept of coronary microembolisation. ${ }^{11}$ Thrombotic and non-thrombotic material ranging in size from 100-550 $\mu \mathrm{m}$ was detected in aortocoronary saphenous vein grafts as well as in native coronary arteries. ${ }^{12}$

Microembolisation in the intracranial cerebral arteries can be detected by transcranial Doppler ultrasound. ${ }^{13}$ Brief, high intensity transient signals (HITS) in the Doppler velocity spectrum correspond to microemboli made of thrombotic and non-thrombotic material in laboratory models. ${ }^{14}{ }^{15}$ In clinical practice HITS have been reported in patients with a variety of potential cardiac sources of microembolism. ${ }^{16-18}$

To investigate coronary microembolisation during PCI further, a direct assessment of microembolisation such as that established for the cerebrovascular circulation would be desirable. We therefore evaluated the feasibility of using a Doppler ultrasound device dedicated to detecting HITS in coronary arteries, firstly in an in vitro model with artificial embolic particles, and subsequently in coronary arteries during PCI. It was our goal to investigate whether intracoronary Doppler can be used to detect coronary microemboli and whether the incidence of microembolism is directly related to the CFVR after PCI.

\section{METHODS}

\section{Criteria for the definition of HITS}

The signals were defined by the following characteristics: high intensity; short duration; unidirectionality within the Doppler velocity spectrum; and being accompanied by a "snap", "chirp", or "moan" on the audible output. The signals were recorded on Super VHS tape and analysed off line for HITS. Two observers had to agree that the signal met the identification criteria of Doppler microembolic signals. ${ }^{19} 20$

The intensity increase of HITS was unidirectional within the Doppler velocity spectrum and maximal over a narrow frequency range (fig 1A, B). The intensity increase of artefact signals was bidirectional and maximal at low frequencies (fig $1 \mathrm{C}, \mathrm{D}$ ).

\section{In vitro experimental setup}

A 0.014 inch guidewire with a $12 \mathrm{MHz}$ pulsed Doppler transducer was connected to a console (FloWire/FloMap System, Volcano Therapeutics, Rancho Cordova, California, USA). The system insonated at a depth of $5.2 \mathrm{~mm}$ and used a 128 point fast Fourier transform to measure the signal intensity. The pulse repetition frequency was set at $100 \mathrm{~Hz}$. The detection threshold for Doppler signals was adjusted to a low intensity and a fast sweep speed (display duration 1.6 seconds) was chosen for the Doppler velocity spectrum.

An experimental open loop system was made of a polyethylene tube with an internal diameter of $3 \mathrm{~mm}$. A flow pattern similar to that in a human coronary artery was obtained by a roller pump (fig 2 ). We positioned a 0.014 inch

Abbreviations: APV, average peak velocity; CFVR, coronary flow velocity reserve; $\mathrm{CK}$, creatine kinase; HITS, high intensity transient signals; $\mathrm{PCl}$, percutaneous coronary intervention 
A

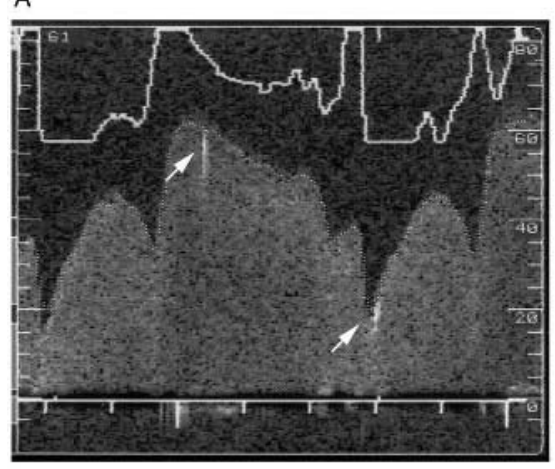

c

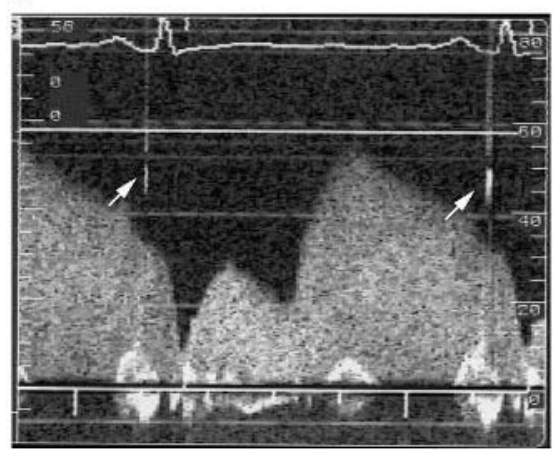

B

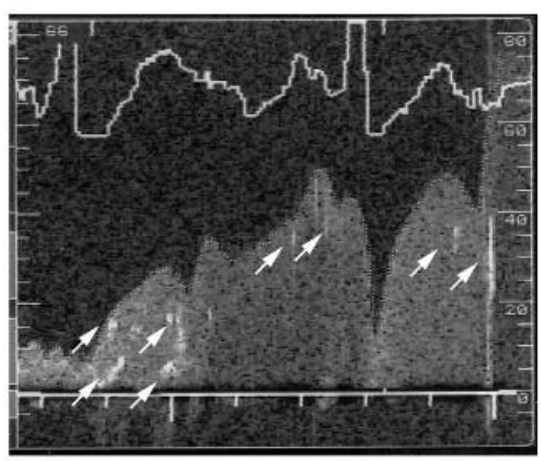

D

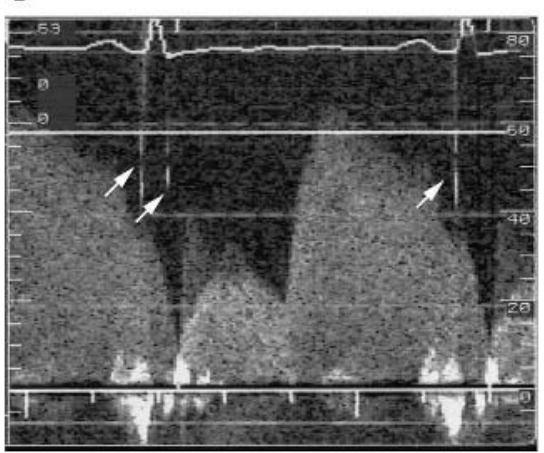

Figure 1 Doppler images during percutaneous coronary interventions in patients. (A) High intensity transient signals (HITS) (arrows) after balloon dilatation. (B) HITS (arrows) after stent implantation. (C, D) Artefact signals (arrows). guidewire with a pulsed Doppler ultrasound transducer mounted on its tip in the flow circuit. The $28^{\circ}$ ultrasound beam provided a sample volume that was $4 \mathrm{~mm}$ from the tip. The sample volume encompassed the central axis of the tube where the velocity should peak. The large sample volume, combined with the FloMap's automatic tracking of the average peak velocity (APV), made the FloWire less position dependent.

The circuit was filled with a starch solution to obtain a blood-like pulsatile flow signal. Sand particles with a mean size of $300 \mu \mathrm{m}$ (range 100-500 $\mu \mathrm{m}$ ) were stirred continuously with the starch solution and randomly released into the circuit. Thus, it was unlikely that an excessive particle load together would result in a lower incidence of particle detection.

The particles were filtered after passing the guidewire and counted under a microscope. The Doppler velocity spectrum was recorded on Super VHS tape and analysed off line for HITS (fig 3A). In another experiment we filled the circuit with water instead of a starch solution. Two different

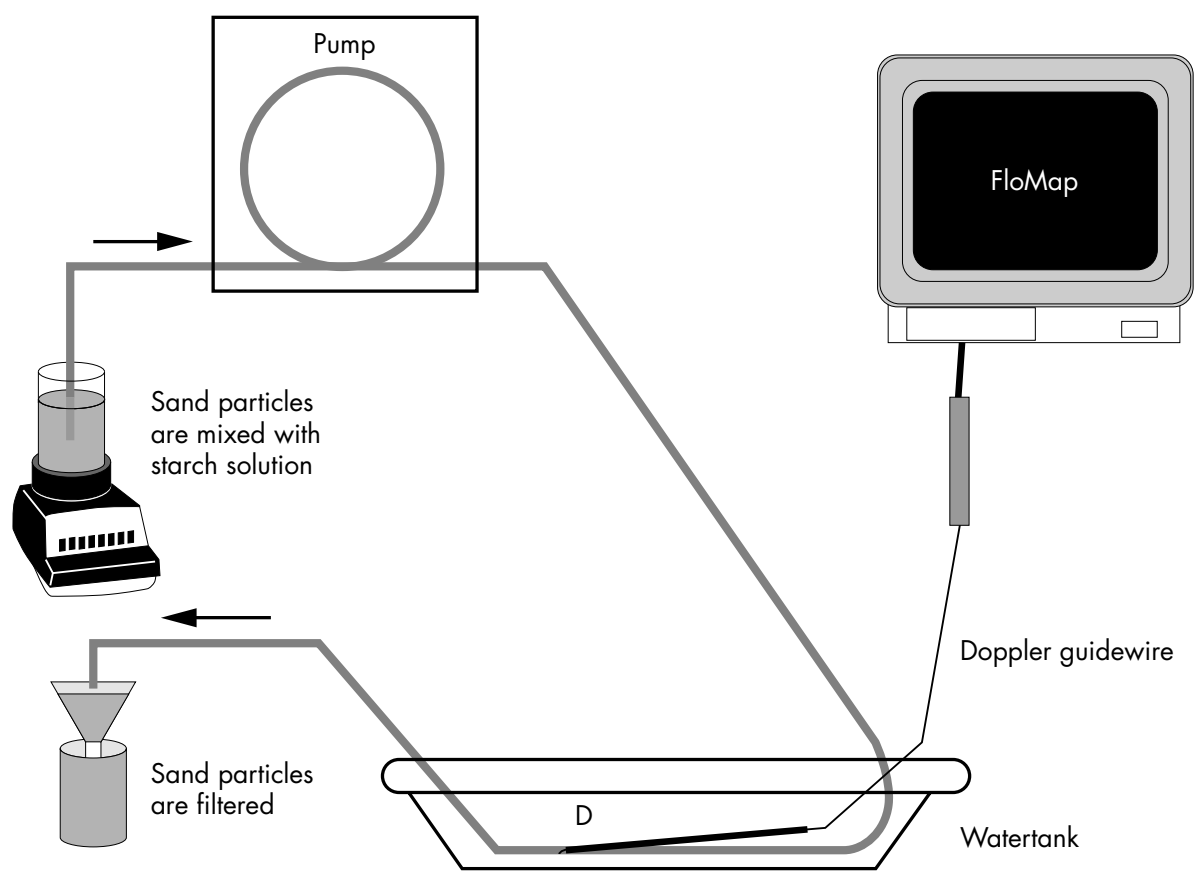

Figure 2 Open loop system used in the in vitro studies. D indicates the tip of the Doppler guidewire. 
A

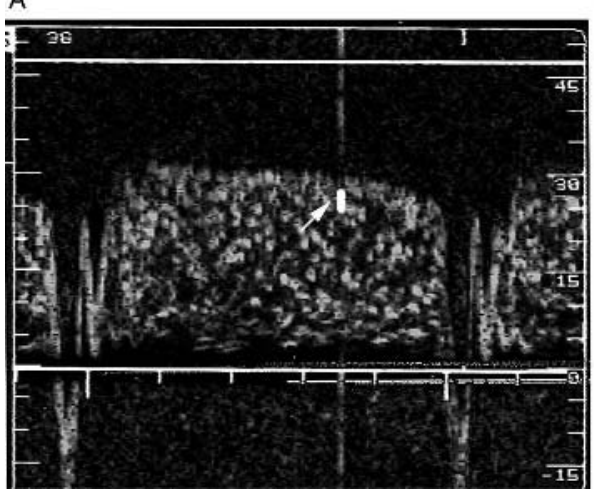

B

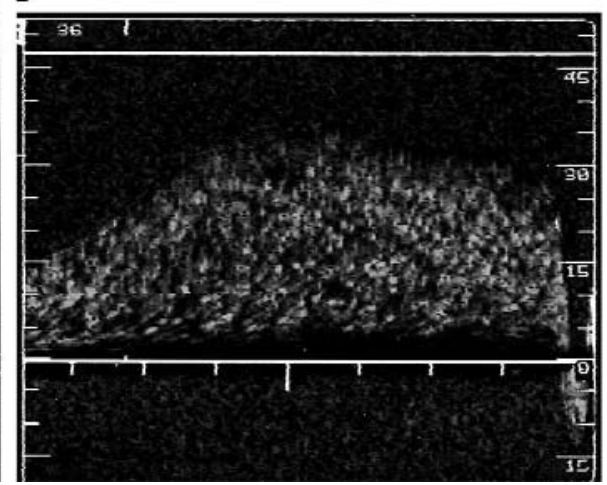

Figure 3 Doppler images in the experimental open loop system with (A) HITS (arrow) representing a sand particle, and (B) without HITS for simulation of excavations. preparations of starch particles were used with a mean particle size of $52 \mu \mathrm{m}$ (range 17-70 $\mu \mathrm{m}$; Sephadex G-25 Superfine, Amersham Pharmacia Biotech, Uppsala, Sweden) and $200 \mu \mathrm{m}$ (range 100-300 $\mu \mathrm{m}$; Sephadex G-50 Medium, Amersham Pharmacia Biotech). About 10 particles were injected into the circuit and passed the guidewire. The Doppler velocity spectrum was recorded on Super VHS tape and analysed off line for HITS detection. We also analysed HITS for a possible relation between relative Doppler intensity and particle size. The Doppler intensity was measured by wavelet analysis of ultrasound signals.

\section{Simulation of balloon deflation}

A second experiment without sand particles was done in the circuit as described above. A balloon catheter with a diameter of $3 \mathrm{~mm}$ was advanced $1-2 \mathrm{~cm}$ proximal to the guidewire. While Doppler recordings were taken on Super VHS tape, balloon deflations were repeated 30 times. Afterwards the tape was analysed for HITS (fig 3B).

\section{Clinical study}

Thirty two consecutive patients (mean (SD) age 61 (11) years; 23 men, nine women) were prospectively studied. They had coronary artery disease and were treated successfully with balloon dilatation, stent implantation, or both for a single vessel stenosis and underwent periprocedural Doppler analysis at the University Clinic of Jena. The institutional ethics committee approved the study protocol and all patients gave written informed consent. The investigation conformed with the principles outlined in the Declaration of Helsinki.

Inclusion criteria were age between 18-80 years and written informed consent. Exclusion criteria were increase of cardiac markers before PCI; acute myocardial infarction during the four weeks before PCI; terminal renal insufficiency, hypothyroidism, or skeletal muscle injury; chronic occlusion, bifurcation lesion, or in-stent restenosis; multivessel intervention; side branch occlusion or prolonged vasospasm; contraindication for antiplatelet medications; and administration of glycoprotein IIb/IIIa receptor antagonists.

All patients received $100 \mathrm{mg}$ acetylsalicylic acid once daily before the procedure and thereafter. A heparin bolus of $10000 \mathrm{U}$ was given after insertion of the arterial sheath. All patients received $300 \mathrm{mg}$ clopidogrel on the day of PCI. Afterwards they received $75 \mathrm{mg}$ clopidogrel once daily for four weeks. All other medication was given at the discretion of the attending cardiologist.

All patients were monitored for 24 hours after PCI. Cardiac troponin I (upper limit of normal $0.1 \mathrm{ng} / \mathrm{ml}$ ) and CK (upper limit of normal for women $1.17 \mu \mathrm{mol} / \mathrm{l} / \mathrm{s}$ or $70.2 \mathrm{U} / \mathrm{l}$ and for men $1.33 \mu \mathrm{mol} / \mathrm{l} / \mathrm{s}$ or $79.8 \mathrm{U} / \mathrm{l}$ ) were assessed between $12-24$ hours.

\section{Measurement of intracoronary Doppler velocity}

The Doppler transducer was set to the same parameters as described above for the in vitro studies. A fast sweep speed (display duration 1.6 seconds) for the Doppler velocity spectrum was chosen and the detection threshold for Doppler signals was set on a low intensity for all patients in this study. Coronary flow velocity after PCI increased due to hyperaemia. Accordingly, we adjusted the Doppler velocity range spectrum to the expected maximum velocity.

Before and during PCI the Doppler wire was positioned 1$2 \mathrm{~cm}$ distal to the stenosis. At this position the APV was recorded. Before and after each interventional step the CFVR was measured as the ratio of maximum APV to baseline APV. Maximum hyperaemia was induced by intracoronary injection of 30-40 $\mu \mathrm{g}$ adenosine. The Doppler recording on Super VHS tape during and three minutes after each PCI was analysed off line for HITS.

\section{Intraobserver and interobserver agreement}

To assess intraobserver agreement one of the observers counted HITS twice on different days. Interobserver agreement was assessed by two observers analysing the Doppler recordings for HITS. All patients were assessed.

\section{Statistical analysis}

Data were expressed as mean (SD). Differences of parameters within a group were evaluated by the paired samples $t$ test. The independent samples $t$ test was used to compare means for two groups. HITS counted in the in vitro studies were compared and intraobserver and interobserver agreement

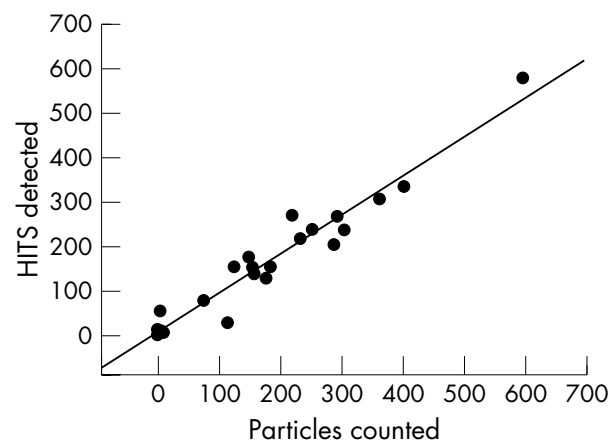

Figure 4 Plot of linear regression analysis showing the relation between the number of particles counted and HITS detected (29 experiments, correlation $r=0.97, p<0.001$ ). 
Table 1 Coronary flow velocity reserve (CFVR) and number of high intensity transient signals (HITS) in 32 patients

\begin{tabular}{|c|c|c|c|c|c|c|c|c|c|c|c|c|}
\hline \multirow[b]{2}{*}{ No } & \multirow[b]{2}{*}{ Sex } & \multirow[b]{2}{*}{ Age (years) } & \multirow[b]{2}{*}{$\begin{array}{l}\text { Target } \\
\text { vessel }\end{array}$} & \multicolumn{3}{|l|}{ CFVR } & \multicolumn{2}{|c|}{ HITS 1st assessment } & \multicolumn{2}{|c|}{ HITS 2nd assessment } & \multicolumn{2}{|c|}{ HITS 2nd observer } \\
\hline & & & & Before BD & After BD & After stenting & After BD & $\begin{array}{l}\text { After } \\
\text { stenting }\end{array}$ & After BD & $\begin{array}{l}\text { After } \\
\text { stenting }\end{array}$ & After BD & $\begin{array}{l}\text { After } \\
\text { stenting }\end{array}$ \\
\hline 1 & $M$ & 64 & RCA & 1.3 & 1.7 & 3.0 & 2 & 14 & 2 & 12 & 3 & 11 \\
\hline 2 & $\mathrm{~F}$ & 53 & RCA & 2.0 & 2.6 & 3.6 & 2 & 16 & 2 & 16 & 5 & 12 \\
\hline 3 & $\mathrm{~F}$ & 63 & RCA & 1.6 & 1.7 & 1.4 & 0 & 9 & 0 & 9 & 0 & 8 \\
\hline 4 & $M$ & 70 & RCA & 2.2 & 2.3 & 2.3 & 1 & 19 & 1 & 20 & 3 & 11 \\
\hline 5 & $M$ & 47 & $\mathrm{RCX}$ & 2.5 & 2.8 & NA & 0 & NA & 0 & NA & 0 & NA \\
\hline 6 & $M$ & 64 & $\mathrm{RCX}$ & 1.4 & 3.0 & NA & 4 & NA & 4 & NA & 2 & NA \\
\hline 7 & $M$ & 53 & LAD & NA & NA & 4.3 & 1 & 5 & 1 & 4 & 6 & 3 \\
\hline 8 & $M$ & 65 & RCA & 1.1 & 1.2 & 2.0 & 0 & 0 & 0 & 0 & 0 & 0 \\
\hline 9 & $M$ & 50 & LAD & 1.5 & 3.0 & NA & 0 & NA & 0 & NA & 0 & NA \\
\hline 10 & $\mathrm{~F}$ & 52 & LAD & 2.4 & 2.2 & 1.8 & 2 & 23 & 3 & 21 & 1 & 23 \\
\hline 11 & $M$ & 75 & RCA & 0.5 & 1.0 & 2.3 & 0 & 18 & 0 & 19 & 0 & 19 \\
\hline 12 & $M$ & 75 & LAD & 2.5 & 2.3 & 2.8 & 1 & 1 & 1 & 1 & 6 & 1 \\
\hline 13 & $M$ & 65 & LAD & 1.0 & 1.3 & 1.4 & 0 & 14 & 0 & 17 & 1 & 16 \\
\hline 14 & $M$ & 54 & LAD & 1.2 & 2.0 & 2.6 & 1 & 2 & 1 & 1 & 1 & 1 \\
\hline 15 & $\mathrm{~F}$ & 65 & LAD & 0.7 & 2.9 & NA & 0 & NA & 0 & NA & 0 & NA \\
\hline 16 & $M$ & 58 & RCA & 1.6 & 2.6 & 2.9 & 4 & 11 & 4 & 10 & 5 & 14 \\
\hline 17 & $\mathrm{~F}$ & 67 & RCA & 1.6 & 1.7 & 3.1 & 0 & 13 & 1 & 12 & 3 & 16 \\
\hline 18 & $M$ & 69 & $\mathrm{RCX}$ & NA & 1.7 & NA & 1 & NA & 1 & NA & 0 & NA \\
\hline 19 & $\mathrm{~F}$ & 75 & RCA & 1.4 & NA & 1.7 & 0 & 10 & 0 & 11 & 0 & 7 \\
\hline 20 & $M$ & 73 & RCA & 3.0 & 3.1 & 2.2 & 20 & 14 & 18 & 13 & 12 & 8 \\
\hline 21 & $M$ & 57 & RCA & 1.5 & 2.8 & NA & 0 & 4 & 0 & 4 & 0 & 3 \\
\hline 22 & $\mathrm{~F}$ & 41 & LAD & 2.0 & 1.6 & 3.4 & 6 & 13 & 5 & 13 & 6 & 15 \\
\hline 23 & $M$ & 78 & LAD & 1.2 & 1.4 & 1.6 & 0 & 0 & 0 & 0 & 0 & 0 \\
\hline 24 & $M$ & 74 & RCA & 2.2 & 1.8 & 1.9 & 1 & 18 & 1 & 19 & 6 & 11 \\
\hline 25 & $M$ & 71 & LAD & 2.2 & 1.9 & 2.1 & 8 & 16 & 7 & 17 & 4 & 7 \\
\hline 26 & $M$ & 62 & LAD & 1.4 & 1.8 & 2.3 & 9 & 0 & 11 & 0 & 5 & 0 \\
\hline 27 & $M$ & 37 & LAD & 1.2 & 2.8 & 2.3 & 1 & 16 & 1 & 17 & 1 & 6 \\
\hline 28 & $M$ & 54 & $\mathrm{RCX}$ & 1.4 & 1.6 & 2.3 & 0 & 12 & 0 & 14 & 0 & 5 \\
\hline 29 & $M$ & 72 & LAD & 1.7 & 1.6 & 2.7 & 0 & 11 & 0 & 13 & 0 & 4 \\
\hline 30 & $\mathrm{~F}$ & 58 & LAD & 0.9 & 1.4 & 2.7 & 4 & 10 & 4 & 9 & 4 & 6 \\
\hline 31 & $\mathrm{~F}$ & 49 & LAD & 1.2 & 1.0 & 3.0 & 2 & 20 & 2 & 18 & 2 & 13 \\
\hline 32 & $M$ & 52 & LAD & 1.4 & NA & 3.0 & 4 & 1 & 4 & 1 & 1 & 0 \\
\hline \multicolumn{2}{|c|}{ Mean (SD) } & 61 (11) & & $1.59(0.58)$ & $2.02(0.65)$ & $2.53(0.66)$ & $2(4)$ & $11(7)$ & $2(4)$ & $11(7)$ & $2(3)$ & $8(6)$ \\
\hline
\end{tabular}

was analysed according to the method of Bland and Altman (plot of difference against mean) and by use of a linear regression analysis expressed as the slope and the correlation coefficient. $^{21}$ All probability values were calculated two sided and a probability level of $\mathrm{p}<0.05$ was considered significant. All calculations were done with SPSS for Windows version 11.5.1 (SPSS Inc, Chicago, Illinois, USA).

\section{RESULTS}

\section{Validation of HITS}

In the artificial coronary circulation model, 3884 (90.4\%) of the 4296 sand particles were correctly detected as emboli and $412(9.6 \%)$ were not detected. Of the 3905 HITS, 3884 $(99.5 \%)$ corresponded to particles and $21 \quad(0.5 \%)$ did not correspond. The detection sensitivity of emboli as HITS was $90 \%$. We found a close linear correlation between particle count and number of HITS in 29 individual experiments $(r=0.97, \mathrm{p}<0.001)$ (fig 4$)$.

We were able to identify Sephadex particles with a mean size of $52 \mu \mathrm{m}$ as HITS. There was no significant correlation between relative Doppler intensity and particle size.

Possible excavations or air bubbles occurring during rapid balloon expansions were simulated by repeated in vitro balloon deflations. We did not detect any HITS during 30 balloon deflations.

\section{Clinical studies}

Balloon dilatation followed by stent implantation for a single vessel stenosis was performed in 27 patients and balloon dilatation alone was done in five patients. Table 1 summarises patient characteristics, the target vessel size, and the results of intracoronary Doppler and CFVR studies. Balloons used for dilatation were significantly smaller than those used for stenting (2.82 (.46) $\mathrm{mm} v 3.19$ (.41) mm, p < 0.001).

\section{Intracoronary Doppler recordings of HITS}

We detected HITS during PCI after 19 (59\%) of 32 balloon inflations and in $24(89 \%)$ of 27 patients after stent implantation. Considering only HITS counts $>2$ as indicators of microembolisation, such incidences were observed in $38 \%$ after balloon deflation and in $82 \%$ after stenting. The number of HITS after stent implantation was significantly higher than after balloon dilatation (11 (7) $\vee 2$ (4), p < 0.001) (table 1). Almost all HITS were observed within 10 seconds after each PCI immediately after balloon deflation. The last HITS occurred about 60 seconds after PCI, but there were too few of these HITS monitored after balloon dilatation and stenting to detect a significant difference.

\section{Intraobserver and interobserver agreement of intracoronary HITS detection}

Correlation between HITS counted on different days by one observer was very high $(r=0.99, \mathrm{p}<0.001)$ (fig 5A) indicating excellent reproducibility for intracoronary Doppler derived HITS. We found a very high correlation coefficient for HITS after balloon dilatation $(r=0.99$, $\mathrm{p}<0.001)$ and stent implantation $(r=0.98, \mathrm{p}<0.001)$. The mean difference of HITS data showed good agreement between the two counts (mean difference -0.13 (0.43), SD (1.19) (95\% confidence interval)) (fig 5B).

Interobserver agreement of HITS count between two blinded observers was good $(r=0.84, \mathrm{p}<0.001)$ (fig 5C). The correlation coefficients for HITS after balloon dilatation $(r=0.75, \mathrm{p}<0.001)$ and after stenting $(r=0.80$, $\mathrm{p}<0.001)$ were high. The mean difference of HITS data 

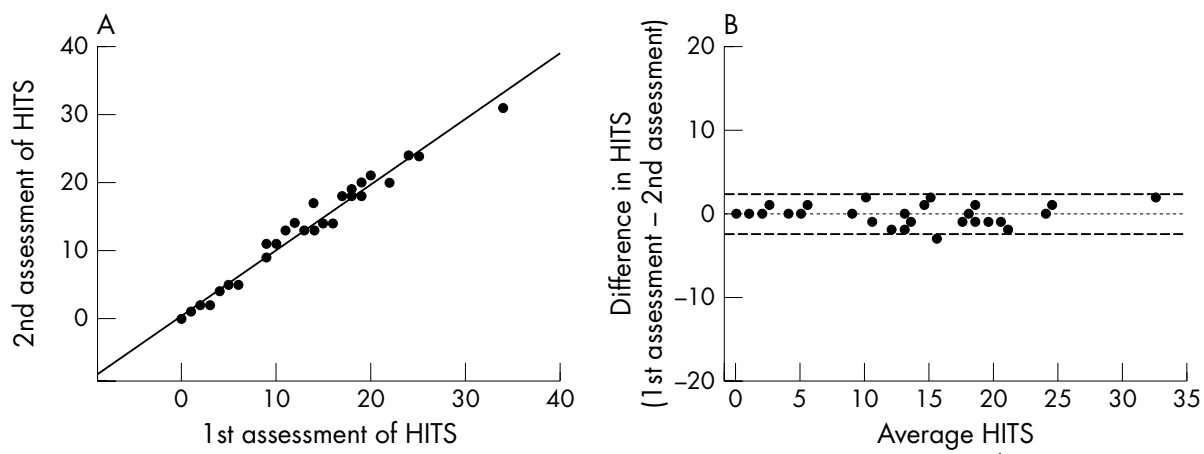

Figure 5 Plots of linear regression analysis and mean difference. (A, B) Relation between the first and second number of HITS counted on different days by the same observer ( $\mathrm{n}=32$, correlation $r=0.99$, $\mathrm{p}<0.001)$. (C, D) Comparison of number of HITS counted by two observers for analysis of interobserver agreement $(\mathrm{n}=32$, correlation $r=0.84, \mathrm{p}<0.001)$
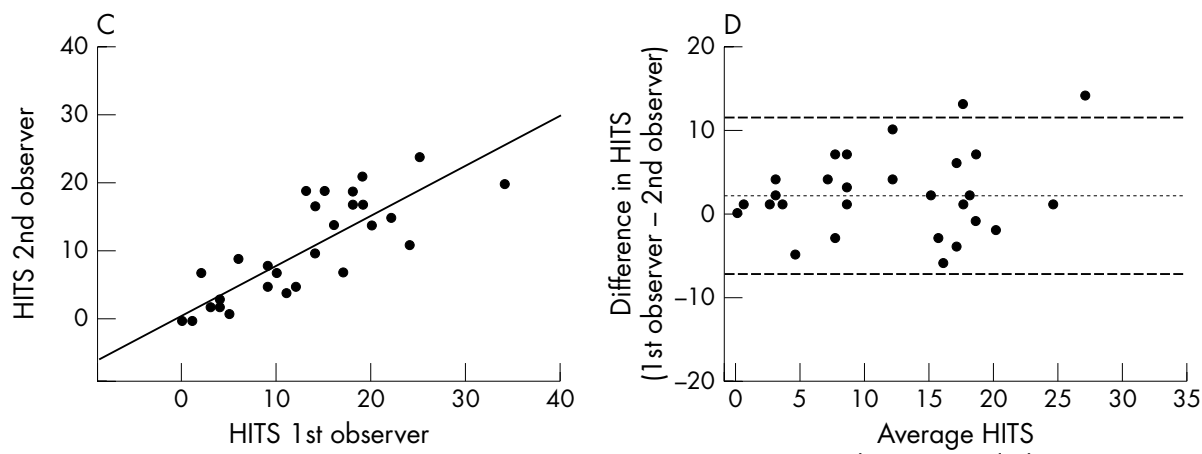

(1st observer; 2nd observer)

showed good agreement between the two measurements (mean difference 2.09 (1.69), SD (4.69), (95\% confidence interval)) (fig 5D).

\section{Comparison of postprocedural CFVR and number of HITS}

In patients with CFVR $<2.0$ baseline APV was significantly higher after PCI than in patients with CFVR $\geqslant 2.0$ (38.7 $(14.9) \mathrm{cm} / \mathrm{s} \vee 24.2(9.5) \mathrm{cm} / \mathrm{s}, \mathrm{p}=0.005)$ (table 2). The number of HITS did not differ significantly between patients with CFVR $<2.0$ and patients with CFVR $\geqslant 2.0$ after balloon dilatation ( 1 (1) $v 3(4)$, not significant) or stent implantation (12 (8) $v 10$ (7), not significant) (fig 6).

Postprocedural CFVR was $<2.0$ in $55 \%$ of all patients after balloon dilatation and in $23 \%$ after stent implantation. Table 1 shows that patients $3,10,20$, and 27 had a reduction in CFVR from balloon dilatation to stent implantation. These seem to

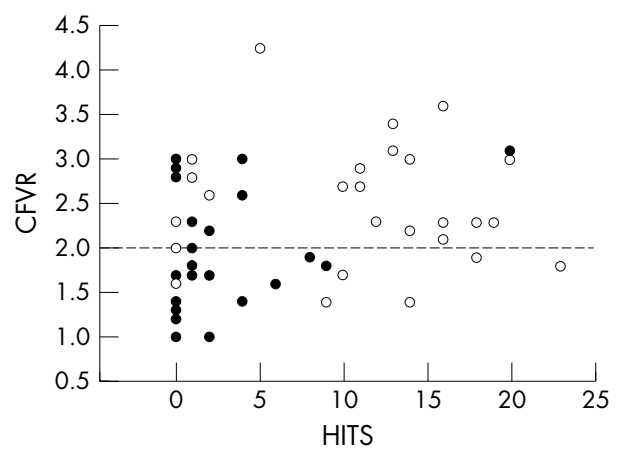

Figure 6 Number of HITS against coronary flow velocity reserve (CFVR) for all patients after balloon dilatation (black dots, correlation $r=0.09$, not significant) and stent implantation (white dots, correlation $r=0.03$, not significant).

\begin{tabular}{|c|c|c|c|c|c|}
\hline Group & $\begin{array}{l}\text { Baseline APV } \\
(\mathrm{cm} / \mathrm{s})\end{array}$ & $\begin{array}{l}\text { Hyperaemic } \\
\text { APV }(\mathrm{cm} / \mathrm{s})\end{array}$ & CFVR & $\begin{array}{l}\text { Heart rate } \\
\text { (beats/min) }\end{array}$ & MAP $(\mathrm{mm} \mathrm{Hg})$ \\
\hline \multicolumn{6}{|l|}{ All patients $(n=32)$} \\
\hline Pre-PCl & $20.8(9.5)$ & $34.7(19.4)$ & $1.59(.58)$ & $72.3(16.2)$ & $106.3(19.5)$ \\
\hline Post-PCl & $27.6(12.4)$ & $58.6(20.8)$ & $2.53(.66)$ & $72.4(11.2)$ & 106.7 (19.7) \\
\hline Post-PCl:pre-PCl & $1.5(.5)$ & $2.2(1.3)$ & $1.8(.9)$ & $1.0(.1)$ & $1.0(.1)$ \\
\hline \multicolumn{6}{|c|}{ Group comparison CFVR $<2.0(n=7)$} \\
\hline Pre-PCl & $23.1(10.3)$ & $38.9(22.9)$ & $1.63(.56)$ & $67.6(18.7)$ & $115.6(22.8)$ \\
\hline Post-PCl & 38.7 (14.9) & $60.6(23.6)$ & $1.64(.19)$ & $76.3(17.9)$ & $115.0(15.9)$ \\
\hline Post-PCl:pre-PCl & $1.9(.6)$ & $2.0(1.0)$ & $1.1(.3)$ & $1.0(.1)$ & $1.0(.1)$ \\
\hline \multicolumn{6}{|c|}{ Group comparison CFVR $\geqslant 2.0(n=25)$} \\
\hline Pre-PCl & $20.3(9.4)$ & $33.8(19.0)$ & $1.58(.59)$ & $73.3(15.9)$ & $104.3(18.6)$ \\
\hline Post-PCl & $24.2(9.5)^{\star \star \star}$ & $58.0(20.4)$ & $2.77(.51)^{\star * * *}$ & $71.5(10.1)$ & $104.9(20.5)$ \\
\hline Post-PCl:pre-PCI & $1.4(.5)^{*}$ & $2.2(1.4)$ & $2.0(.9)^{* *}$ & $0.9(.2)$ & $1.0(.1)$ \\
\hline \multicolumn{6}{|c|}{$\begin{array}{l}\text { Values are mean }(S D) \text {. } \\
{ }^{*} p=0.023 ;{ }^{* *} p=0.021 ;{ }^{* * *} p=0.005 ;{ }^{* * * *} p<0.001 \text { versus patient group with } C F V R<2.0 \text {. } \\
\text { APV, average peak velocity; MAP, mean arterial pressure. }\end{array}$} \\
\hline
\end{tabular}


be the ideal cases to show the presence of distal embolisation, since no other explanations would clarify why, with a restored lumen patency, CFVR decreased. In fact, three of these patients had a greater than average number $(>11)$ of HITS and patient 10 had the highest number (23) of HITS recorded during the first post-stenting assessment. Eleven patients with no improvement of CFVR after stenting compared with balloon angioplasty or who did not reach a CFVR $>2.0$ after stenting tended to have more HITS than did 15 patients with an increased CFVR after stenting (13.7 (6.1) $v 9.0(6.9), \mathrm{p}=0.08)$.

\section{Comparison of number of HITS and cardiac markers}

Overall, the incidence of a rise in cardiac troponin I to more than twice the upper limit of normal was $18.8 \%$. None of the patients experienced a CK increase to more than twice the upper limit of normal. We did not find a significant difference in the number of HITS between patients with and patients without a cardiac troponin I increase to more than twice the upper limit of normal (10 (9) $v 12$ (9), not significant).

\section{DISCUSSION}

We have shown in this study for the first time that a commercially available intracoronary Doppler ultrasound device can be used to detect HITS in the coronary circulation. This promises to be a novel approach to evaluate the incidence of coronary microembolisation during coronary interventions.

\section{Detection of HITS by intracoronary Doppler ultrasound}

The high sensitivity of Doppler ultrasound in detecting emboli is shown by the ability to detect particles in the in vitro experimental setup of approximate diameters between 52-300 $\mu \mathrm{m}$. The emboli found in patients after sudden cardiac death after PCI or thrombolytic administration had an average size of $250 \mu \mathrm{m} .{ }^{22}$ Therefore, the intracoronary Doppler would be able to detect emboli of a size sufficient to block small to medium sized intramural coronary artery branches in vivo.

In the cerebrovascular circulation the sensitivity for detecting HITS is similar to our findings. ${ }^{15}$ The smallest emboli that were assessed experimentally were 200 to $400 \mu \mathrm{m}$ in diameter. ${ }^{15}$ The minimum detection limit of the transcranial Doppler is unknown. ${ }^{13}$ In contrast to transcranial Doppler the high frequency transducer of the intracoronary Doppler provides a higher resolution at the cost of a reduced depth of penetration. Therefore, we were able to identify particles with a mean size of $52 \mu \mathrm{m}$ as HITS.

We did not use the algorithm applied for intracerebral HITS detection, as we cannot assess the particles at different distances from the ultrasound sensor. Therefore, the differentiation of HITS from artefacts requires clearly defined criteria. These criteria were easily applicable in both the in vitro and in vivo studies. Usually the amplitude of a Doppler microembolic signal is at least $3 \mathrm{~dB}$ higher than that of the background flow signal. ${ }^{19}$ We did not use an intensity cut off for HITS detection, which is frequently applied in the cerebral circulation. ${ }^{23}$ In this first application of HITS detection in the coronary circulation we were interested in the complete intensity spectrum, as we did not yet know what signals to expect during a PCI. Therefore, Doppler speckle may have been mistaken for rare HITS at low frequencies in our study, but the good interobserver agreement suggests a good reproducibility. ${ }^{13}$

\section{HITS during coronary interventions}

We observed a significantly higher number of HITS after stent implantation than after balloon dilatation. After deflation of the predilatation balloon we observed HITS in only one third of lesions, whereas after deflation of the stent balloon after stent placement we observed HITS in about $82 \%$, and in most cases a considerable number. This can be explained by the different mechanisms of balloon angioplasty and stenting. Balloon angioplasty enlarges the lumen by local dissection, whereas stenting works through plaque compression. ${ }^{24}$ Stone $e t ~ a l^{8}$ found an enzyme increase after stenting more often than after balloon dilatation alone, and they assumed that this was caused by microembolisation. Also, the smaller balloon sizes for pre-dilatation before stenting must be taken into account for the lower incidence of HITS with balloon dilatation.

Herrmann et $a l^{25}$ reported an association between a periprocedural enzyme rise and a reduced CFVR after stenting in patients with stable angina pectoris. They assumed that microembolisation probably reduced the CFVR. The high incidence of an impaired CFVR was caused by an increased baseline APV after PCI. These findings were similar to those of our study. Herrmann et $a^{25}$ related the increase in baseline APV to minor forms of embolic myocardial injury. We observed a trend of a higher incidence of HITS immediately after stenting in patients who did not have improved CFVR than after balloon angioplasty, during which in general the incidence of HITS was much lower. A negative impact of microembolisation on CFVR after stenting would probably be counterbalanced by a greater luminal cross section after stent placement than after balloon angioplasty. ${ }^{26}$

We did not, however, observe a close relation between the incidence of HITS and a cardiac marker enzyme increase. The extent of myocyte damage may be related not to the number but to the size of emboli. It is also conceivable that many patients experience microembolisation but do not go on to myonecrosis either because the embolic burden is small or because adaptive responses accommodate the process. ${ }^{10}$

The discrepancy and lack of close correlation between enzymatic detection of myocardial damage and microembolisation was also observed during rotablation. This interventional method has a known incidence of peripheral embolisation, but intraprocedural perfusion defects were detected despite a lack of enzymatic evidence of myocardial necrosis. ${ }^{27}$ There is further evidence that the incidence of emboli and obstruction of peripheral arterioles alone may not determine a clinically adverse response. Experimental studies have shown that coronary microembolisation induced a progressive reduction in regional function without a measurable decrease in regional flow, but that it was associated with an inflammatory response. ${ }^{28} 29$ The individual response to embolic events in the coronary system may decide whether the regularly observed microembolisation during a PCI leads to a clinical consequence.

\section{Limitations}

The size and composition of an individual embolus cannot be derived from its signal intensity in the Doppler spectrum. ${ }^{13}$ The size of a single embolus may, however, determine its clinical consequences. The method of detecting HITS also cannot differentiate between air bubbles and solid materials. In the experimental setting, however, for simulation of excavations we did not find HITS after repeated balloon deflations. In a recently conducted clinical study, the glycoprotein IIb/IIIa receptor antagonist tirofiban reversibly suppressed HITS at full dosage in the cerebrovascular circulation. These findings suggest that emboli of arterial origin are solid. ${ }^{30}$ Therefore, we conclude that air embolism during PCI is unlikely to account for HITS in our study.

Despite the evidence that we can detect microemboli with this new intracoronary approach, there was no close relation 
between clinical events and the number of HITS. We had included patients with stable angina to allow a standardised experimental protocol and enough time for Doppler registration during this first application of the new method. Future studies will require a larger number of patients in higher risk populations to examine a possible closer relation between HITS and clinical end points.

\section{Clinical implications}

The detection of coronary emboli during PCI with an intracoronary Doppler ultrasound device is feasible. Coronary microembolism is often induced during PCI, especially after stent implantation. Even though no correlation was found between HITS and CFVR or cardiac marker enzyme increase after PCI, the number of HITS can serve as an indicator to discriminate procedures with different embolic risks. HITS detection may be useful in the evaluation of treatment modalities for the reduction of peri-interventional microembolism in high risk patients.

\section{Authors' affiliations}

P Bahrmann, H R Figulla, M Ferrari, G S Werner, Clinic of Internal Medicine I, Friedrich-Schiller University, Jena, Germany

M Wagner, A Voss, Department of Medical Engineering, University of Applied Sciences, Jena, Germany

\section{REFERENCES}

1 Topol EJ, Leya F, Pinkerton CA, for the CAVEAT Study Group, et al. A comparison of coronary angioplasty with directional atherectomy in patients with coronary artery disease. N Engl J Med 1993;329:221-7.

2 Califf RM, Abdelmeguid AE, Kuntz RE, et al. Myonecrosis after revascularisation procedures. J Am Coll Cardiol 1998;31:241-51.

3 Brener SJ, Ellis SG, Schneider J, et al. Frequency and long-term impact of myonecrosis after coronary stenting. Eur Heart J 2002;23:869-76.

4 Abdelmeguid AE, Topol EJ, Whitlow PL, et al. Significance of mild transient release of creatine kinase-MB fraction after percutaneous coronary interventions. Circulation 1996;94:1528-36.

5 Ravklide J, Nissen H, Mickley H, et al. Cardiac troponin T and CK-MB mass release after visually successful percutaneous transluminal coronary angioplasty in stable angina pectoris. Am Heart J 1994;127:13-20.

6 Johansen O, Brekke M, Stromme JH, et al. Myocardial damage during percutaneous transluminal coronary angioplasty as evidenced by troponin $T$ measurements. Eur Heart J 1998;19:112-7.

7 Reimers B, Lachin M, Cacciovillani L, et al. Troponin T, creatine kinase MB mass, and creatine kinase $M B$ isoform ratio in the detection of myocardial damage during non-surgical coronary revascularization. Int J Cardiol 1997;60:7-13

8 Stone GW, Mehran R, Dangas G, et al. Differential impact on survival of electrocardiographic $Q$-wave versus enzymatic myocardial infarction after percutaneous intervention: a device-specific analysis of 7147 patients. Circulation 2001;104:642-7
9 Kong TQ, Davidson CJ, Meyers SN, et al. Prognostic implication of creatine kinase elevation following elective coronary artery interventions. JAMA 1997;277:461-6.

10 Topol EJ, Yadav, JS. Recognition of the importance of embolization in atherosclerotic vascular disease. Circulation 2000;101:570-80.

11 Erbel R, Heusch G. Coronary microembolization. J Am Coll Cardiol 2000;36:22-4.

12 Carlino M, De Gregorio J, Di Mario C, et al. Prevention of distal embolization during saphenous vein graft lesion angioplasty: experience with a new temporary occlusion and aspiration system. Circulation 1999;99:3221-3.

13 Markus HS. Monitoring embolism in real time. Circulation 2000;102:826-8.

14 Russell D, Madden KP, Clark WM, et al. Detection of arterial emboli using Doppler ultrasound in rabbits. Stroke 1991;22:253-8.

15 Markus HS, Tegeler C. Experimental aspects of high-intensity transient signals in the detection of emboli. J Clin Ultrasound 1995;23:81-7.

16 Pettelot G, Bracco J, Gibelin P, et al. Detection of embolic signals using Doppler ultrasound: a new approach to cardiac embolism. Int J Cardiol 1997; 58: 1-5

17 Stygall J, Kong R, Walker JM, et al. Cerebral microembolism detected by transcranial Doppler during cardiac procedures. Stroke 2000:31:2508-10

18 Eicke BM, Barth V, Kukowski B, et al. Cardiac microembolism: prevalence and clinical outcome. J Neurol Sci 1996;136:143-7.

19 Anon. Consensus Committee of the Ninth International Cerebral Hemodynamic Symposium. Basic identification criteria of Doppler microembolic signals. Stroke 1995;26:1123.

20 Ringelstein EB, Droste DW, Babikian VL, et al. Consensus on microembolus detection by TCD. Stroke 1998;29:725-9.

21 Bland JM, Altman DG. Statistical methods for assessing agreement between two methods of clinical measurement. Lancet, 1986;i, 307-10.

22 Saber RS, Edwards WD, Bailey KR, et al. Coronary embolization after balloon angioplasty or thrombolytic therapy: an autopsy study of 32 cases. J Am Coll Cardiol 1993:22:1283-8.

23 Markus HS, Ackerstaff R, Babikian VL, for the International Consensus Group on Microembolus Detection, et al. Consensus on microembolus detection by TCD. Stroke 1998;29:725-9.

24 Ahmed J, Mintz G, Weissman NJ, et al. Mechanism of lumen enlargement during intracoronary stent implantation: an intravascular ultrasound study. Circulation 2000;102:107-10.

25 Herrmann J, Haude M, Lerman A, et al. Abnormal coronary flow velocity reserve after coronary intervention is associated with cardiac marker elevation. Circulation 2001; 103:2339-45.

26 Kern MJ, Dupouy P, Drury JH, et al. Role of coronary artery lumen enlargement in improving coronary blood flow after balloon angioplasty and stenting: a combined intravascular ultrasound Doppler flow and imaging study. J Am Coll Cardiol 1997;29:1520-7.

27 Koch KC, vom Dahl J, Kleinhans E, et al. Influence of a platelet GPIlb/Illa receptor antagonist on myocardial hypoperfusion during rotational atherectomy as assessed by myocardial Tc-99m sestamibi scintigraphy. J Am Coll Cardiol 1999;33:998-1004

28 Dörge $H$, Neumann T, Behrends $M$, et al. Perfusion-contraction mismatch with coronary microvascular obstruction: role of inflammation. Am J Physiol Heart Circ Physiol 2000;279:H2587-92.

29 Thielmann M, Dörge H, Martin C, et al. Myocardial dysfunction with coronary microembolization: signal transduction through a sequence of nitric oxide, tumor necrosis factor- $\alpha$, and shingosine. Circ Res 2002;90:807-13.

30 Junghans U, Siebler $M$. Cerebral microembolism is blocked by tirofiban, a selective nonpeptide platelet gylcoprotein Ilb/llla receptor antagonist. Circulation 2003; 107:2717-21. 